\title{
PRODUÇÃO INTELECTUAL DOCENTE DO INSTITUTO DE MATEMÁTICA/UFRGS
}

\section{PRODUCCIÓN INTELECTUAL DE LOS PROFESORES DEL INSTITUTO DE MATEMÁTICA/UFRGS}

\author{
Daniela Gralha de Caneda Queiroz * \\ Ana Maria Mielniczuk de Moura ***
}

\begin{abstract}
RESUMO:
Introdução: Esse estudo é uma pesquisa bibliométrica, cujo objetivo é avaliar a produção docente do Departamento de Estatística (DEST) e do Departamento de Matemática Pura e Aplicada (DMPA) do Instituto de Matemática (IM) da Universidade Federal do Rio Grande do Sul (UFRGS), em relação aos artigos de periódicos publicados eletronicamente, entre 2007 e 2011. Objetivo: São objetivos específicos desse estudo: analisar a produção científica dos docentes no período, verificar o Qualis e a nacionalidade dos periódicos e avaliar o nível de colaboração entre os autores e as instituições. Metodologia: É um estudo bibliométrico de caráter descritivo, sob forma de levantamento, que teve suas respostas analisadas na figura de quadros, tabelas, gráficos e grafos. Resultados: Apresenta como resultados a produtividade de 177 artigos, sendo a produção do DEST (64,4\%) superior a do DMPA (35,6\%). Mostra que os Qualis dos periódicos analisados possuem, em sua maioria, Qualis B1 $(27,11 \%)$, B2 $(16,38 \%)$ e A2 $(15,81 \%)$. Constata que os periódicos são em sua maioria estrangeiros $(62 \%)$, em detrimento dos nacionais $(38 \%)$. Conclusões: Expõe que os docentes do IM possuem elevado nível de colaboração, constituindo uma rede densa de relacionamento. Explana que há um bom nível de colaboração interinstitucional, na sua maioria com instituições nacionais. Sugerem-se novos estudos qualitativos para o encontro de respostas de questões que ficaram em aberto.
\end{abstract}

Palavras-chave: Bibliometria. Produção docente. Periódicos. Coautoria. Colaboração interinstitucional.

Em um tempo em que cada vez mais se vive a sociedade do conhecimento, o nível de desenvolvimento de um país pode ser medido, entre outros quesitos, pela sua produção em pesquisa científica. No Brasil, a pesquisa científica é maciçamente realizada pelo setor público (através de *Mestranda em Comunicação e Informação pela UFRGS. E-mail: daniela.caneda@ufrgs.br **Doutora em Comunicação e Informação. Professora Programa de Pós Graduação em Comunicação e Informação (PPGCOM/UFRGS). E-mail: ana.moura@ufrgs.br 
fundações, hospitais e universidades), que recebe investimentos na ordem de 1,1\% do nosso PIB (aproximadamente, 23 bilhões de dólares). Nas últimas décadas a pesquisa científica brasileira deu um salto de crescimento, com aumento no número de doutores formados e de artigos científicos produzidos, o que já fez o país ocupar no passado o $14^{\circ}$ lugar no ranking de publicações de artigos científicos (CRUZ, 2013).

Meneghini (2009) argumenta que esse aumento na produção científica deve-se à política nacional de fomento brasileira, representada pelo CNPq (Conselho Nacional de Desenvolvimento Científico e Tecnológico) e pela CAPES (Coordenação de Aperfeiçoamento de Pessoal de Nível Superior). Além dessas duas instituições, o autor defende que o programa SciELO (que indexa os melhores periódicos brasileiros), com o apoio da FAPESP (Fundação de Amparo à Pesquisa do Estado de São Paulo) e da BIREME (Biblioteca Virtual em Saúde), incrementou a qualidade dos periódicos nacionais.

No caso das universidades, essa informação pode ser verificada através da produção intelectual docente, que tem confirmado o papel das universidades como fonte de informação e multiplicadora de conhecimento. A produção intelectual docente, além de trazer visibilidade às universidades, traz, em consequência, mais investimentos, que se traduzem em mais verbas para projetos de pesquisa e extensão. Ademais, a produção intelectual, quando devidamente registrada, serve também como memória da instituição.

Como bem coloca Maltrás Barba (2003), este tipo de avaliação permite saber quem produz resultados em determinado período e permite também compreender a constituição e o grau de maturidade de determinada instituição científica. Neste sentido é que convergiu essa pesquisa, como parte de pesquisa maior de mestrado, aplicado no Instituto de Matemática (IM), da Universidade Federal do Rio Grande do Sul (UFRGS), em que se investigou: quais as características da produção intelectual docente nos artigos de periódicos publicados entre os anos de 2007 e 2011? Para tanto, analisou-se a produtividade docente; verificou-se o Qualis e a nacionalidade dos periódicos; avaliou-se o nível de colaboração entre autores e instituições. 
O IM, atualmente Instituto de Matemática e Estatística (IME), formado pelos Departamentos de Estatística (DEST) e de Matemática Pura e Aplicada (DMPA), contava, à época dessa pesquisa, com 89 professores, os quais atendiam, aproximadamente, 16 mil matrículas de alunos, distribuídos em 400 turmas de 150 disciplinas de diversos cursos. O IM possui também três programas de pós-graduação (todos nota 4 na Avaliação Trienal 2013 da Capes) - Ensino de Matemática, Matemática e Matemática Aplicada - os quais atendem, aproximadamente, 130 alunos.

\section{MATERIAIS E MÉTODOS}

Foi aplicada pesquisa do tipo bibliométrica, descritiva, sob forma de levantamento. O corpus da pesquisa foi composto pela totalidade dos artigos produzidos pelos docentes do IM/UFRGS, entre os anos de 2007 e 2011, catalogados no SABi (Sistema de Automação de Bibliotecas) da UFRGS e disponibilizados eletronicamente. Para cada artigo foram coletados: número de sistema; autoria; instituições aos quais os demais autores estavam vinculados nos artigos com múltipla autoria; nome e nacionalidade do periódico; e ano de publicação do artigo. Os dados coletados foram tabulados no programa Microsoft Office Excel 2007; em um segundo momento utilizaram-se os softwares BibExcel, para análise dos dados, e UCINET e NETDRAW, para visualização das redes de colaboração.

\section{RESULTADOS E DISCUSSÃO}

A seguir são apresentados e analisados os resultados obtidos referentes à produtividade docente, periódicos e Qualis, nacionalidade dos periódicos, colaboração entre autores e colaboração interinstitucional.

\subsection{Produtividade Docente}

O número total de artigos produzidos individualmente pelos docentes foi de 214 , sendo que 32 possuíam dois ou mais autores do mesmo departamento do IM, totalizando 177 artigos. A produção dos artigos foi feita por 53 (59, 5\%) 
docentes dos departamentos (11 do DEST e 25 do DMPA). Destes, 5 foram responsáveis por $42 \%$ da produção, enquanto que, no outro extremo, 19 foram responsáveis por apenas $8,88 \%$, fazendo-se presente a Lei do Elitismo de Price (ARAÚJO, 2006, p. 14).

O DEST foi responsável por $64,4 \%$ (114 artigos) da produção, enquanto que o DMPA, por $35,6 \%$ (63), apesar do número de docentes produtores de artigos do DEST (17 professores) ser menor do que o do DMPA (36 professores). Acredita-se que o motivo seja porque o DEST trabalha mais junto a outras áreas da ciência (epidemiologia, genética, ecologia, entre outras), sugerindo uma forte colaboração interdepartamental, que se traduz na própria produção científica, como muito bem explicitado por Vanz (2009). Essa produção de artigos por departamento apresentada no Gráfico ${ }^{1}{ }^{1}$, encontra-se assim distribuída por cada ano analisado:

Gráfico 1 - Produção de artigos por departamentos do IM/UFRGS por ano.

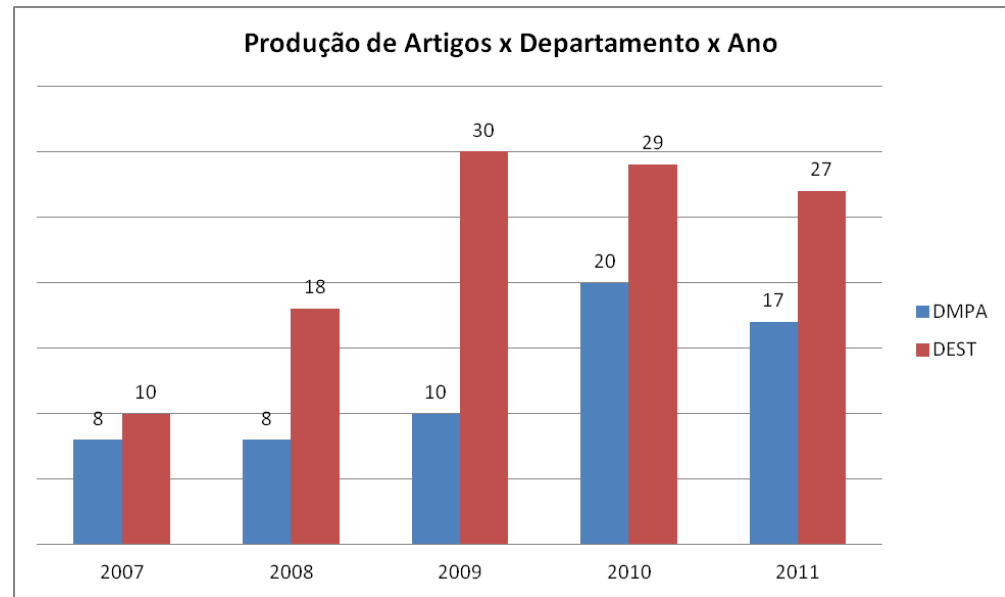

Fonte: Resultado da pesquisa

Os três últimos anos analisados foram os mais produtivos, sendo o ano de 2010 de maior produção dos docentes. Acredita-se que a produtividade 
docente tenha dado esse salto em virtude da chegada de novos docentes através do REUNI ${ }^{2}$, em que o IM recebeu 18 novos professores.

Segundo Cunha (2012), ex-diretor do IM, artigos na área da matemática são mais difíceis de publicar, pois não são interdisciplinares, e porque o tempo entre submissão e publicação do artigo é mais longo. Estudos fornecem subsídios para entender esse comportamento da área de matemática, apontando que "a matemática, em comparação com a maioria das outras ciências, é menos propensa ao trabalho em equipe" (MEADOWS, 1999, p. 108). A produtividade docente analisada aqui será, a seguir, correlacionada com a qualidade dos periódicos em que os artigos foram publicados.

\subsection{Periódicos}

Os 177 artigos foram publicados em 125 diferentes periódicos (em 30 deles houve a publicação de mais de um artigo), denotando-se uma alta diversidade das publicações. Observou-se que o maior número de periódicos possuía Qualis ${ }^{34}$ B1, seguido do B2, A2, B3, A1, B4, B5 e NI (para aqueles periódicos sem Qualis). $70 \%$ dos artigos produzidos no período foram publicados em periódicos com Qualis que variam de A1 a B2, indicando a alta qualidade das publicações do IM.

2 Programa de Apoio a Planos de Reestruturação e Expansão das Universidades Federais, cujo objetivo é o de "criar condições para a ampliação do acesso e permanência na educação superior, no nível de graduação" (BRASIL, 2007).

${ }^{3}$ Esse indicador refere-se ao indicativo de qualidade dos periódicos adotado pela CAPES. Os indicadores são A1, A2, B1, B2, B3, B4, B5 e C, sendo o indicador A1 o de maior qualidade e o C, com peso zero.

${ }^{4}$ A coleta das informações referentes ao Qualis dos periódicos foi realizada na última semana do mês de abril de 2012. Importante destacar que os periódicos sofreram avaliação e nova classificação após a coleta dos dados, ainda no primeiro semestre do ano de 2012. 
Em relação ao fator de impacto ${ }^{5}$ somente 85 (68\%) possuíam tal avaliação. Os periódicos com fatores de impacto mais altos foram aqueles que publicaram artigos de docentes vinculados ao DEST. Contudo, "artigos sobre ciências exatas, [...] têm menor densidade de citações que aqueles ligados às ciências da saúde" (THOMAZ; ASSAD; MOREIRA, 2011), explicando porque os periódicos da área da saúde têm fator de impacto maior que o das ciências exatas. Entre os 11 docentes que mais publicaram em periódicos com alto Qualis, 7 estão entre os que mais produziram artigos. O Gráfico 2 abaixo mostra a distribuição desses periódicos por ano:

Gráfico 2 - Número de periódicos por Qualis conforme o ano.

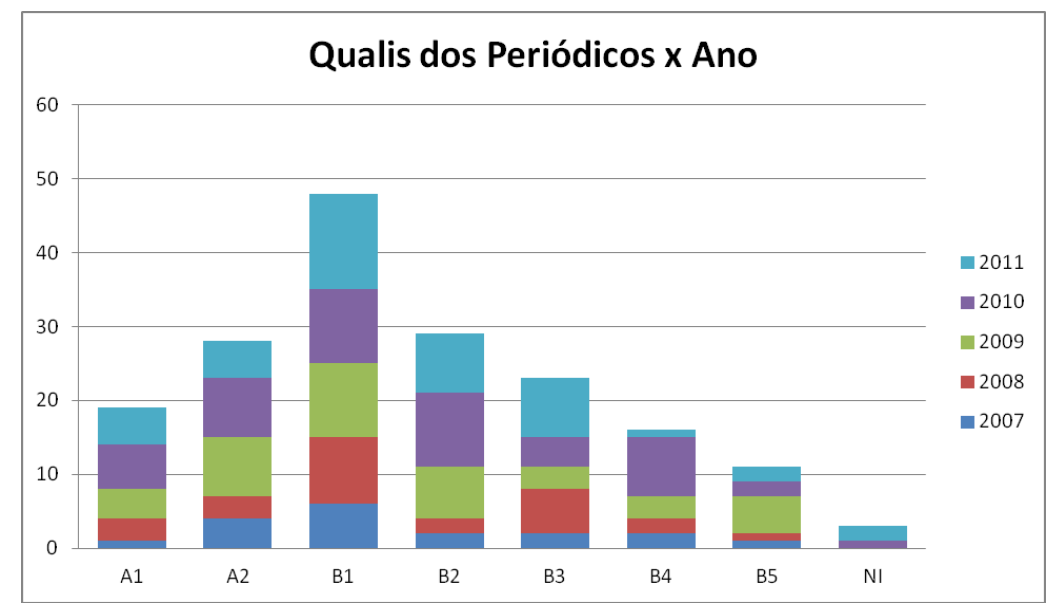

Fonte: Resultado da pesquisa

No âmbito geral, percebeu-se que houve um aumento na qualidade dos periódicos em que os docentes do IM publicam seus artigos a partir do ano de 2009. Uma hipótese para esse cenário seria a busca pelo reconhecimento por parte dos docentes em questão, como bem coloca Maltrás-Barba (2003),

\footnotetext{
${ }^{5}$ Fator de Impacto "é um sistema que determina a quantidade de vezes que uma publicação é citada em certo período de tempo, dividida pela quantidade de artigos publicados nesse mesmo período. A Thomson Reuters (antigo Institute for Scientific Information, mais conhecido como ISI) utiliza nessa avaliação um período de dois anos. O Fator de Impacto é publicado no Journal Citation Report (JCR)." (UNIVERSIDADE..., 2012, online)
} 
através da melhoria da qualidade das pesquisas desenvolvidas. Outra hipótese talvez seja também o aumento no número de docentes, como já descrito.

Entre os periódicos, 62\% (110) são estrangeiros e 38\% (67), nacionais. A explicação pode ser encontrada nos estudos de Vanz (2009):

Na literatura internacional, a Matemática é caracterizada pela baixa colaboração nacional e alta colaboração internacional, atribuída ao tamanho da área, que, por ser pequena, incentiva os pesquisadores a buscar parceiros na comunidade internacional. (VANZ, 2009, p.152).

Como $73,9 \%$ dos coautores dos artigos publicados são vinculados a instituições nacionais, induz-se que os pesquisadores do Brasil estão conseguindo publicar a pesquisa brasileira em periódicos estrangeiros. Os periódicos que mais publicaram artigos dos docentes do IM são do Brasil, seguidos dos Estados Unidos, Holanda e Inglaterra, sendo que os periódicos com fator de impacto mais elevado não são nacionais.

Os periódicos brasileiros foram os que mais publicaram artigos dos docentes do IM, seguido dos periódicos americanos e holandeses. A partir do ano de 2009 aumentou a publicação em periódicos estrangeiros, indicando uma internacionalização da pesquisa produzida pelos docentes do IM.

\subsection{Colaboração entre os Autores}

Somente 8 artigos $(4,5 \%)$, entre 177 , foram escritos somente por um único autor (autoria individual). Predominam os artigos escritos por três, dois e quatro autores, respectivamente, totalizando $58 \%$ dos artigos (Gráfico 3). A coautoria dual (dois autores) aparece em 19,2\% dos artigos e a coautoria múltipla (mais de dois autores), em 76,3\%. Cabe aqui uma deferência ao trabalho de Vanz (2009, p. 171), em que coloca que a "pesquisa em Matemática, [...] se desenvolve por autores isolados ou grupos de dois ou três pesquisadores". Chama a atenção artigo publicado por 66 autores. Contudo, tal artigo trata do assunto astronomia (física), área em que comumente há uma "alta concentração de autores nas pesquisas" (VANZ, 2009, p.171). Meadows (1999) também reforça essa ideia, escrevendo a respeito do crescimento do 
trabalho em equipe na área da física, que tem acarretado muitos nomes de autores em uma só publicação.

A seguir é apresentado um gráfico em que esse número de autores por artigo é distribuído conforme os anos do período da pesquisa. Percebe-se que entre os anos de 2009 a 2011 houve uma grande produção de artigos com 2 a 4 autores. Aqui se pode inferir que a produtividade dos docentes do IM aumentou do ano de 2009 para cá porque cresceu o número de colaborações entre os pesquisadores. "A coautoria é um fenômeno indicativo da colaboração" (MALTRÁS BARBA, 2003, p. 247).

Gráfico 3 - Número de autores por artigo conforme o ano

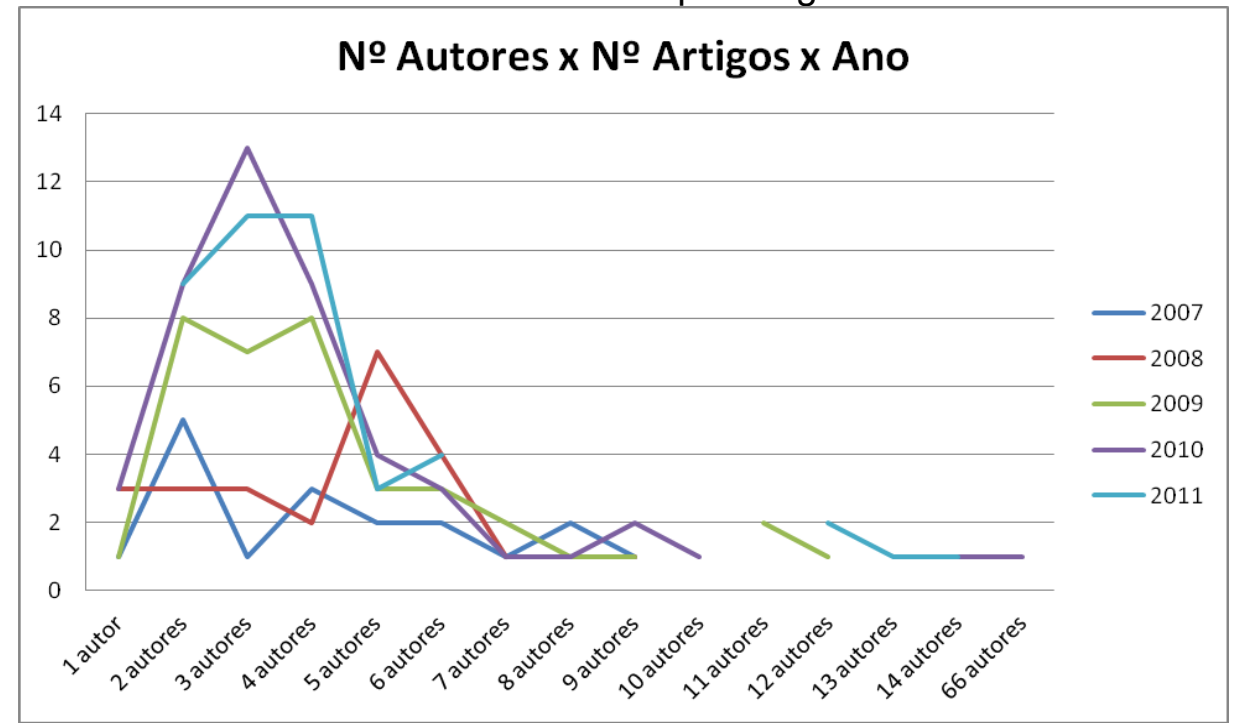

Fonte: Resultado da pesquisa.

Nos 177 artigos analisados, publicados em 125 periódicos, encontrou-se 530 autores, sendo 53 deles pertencentes ao IM (17 do DEST e 36 do DMPA, como já colocado). A relação entre esses autores resultou em um grafo com 509 nós e 7730 laços, indicando uma rede extremamente densa. Em virtude dessa densidade e para fins de facilidade de análise, optou-se por trabalhar com todos aqueles docentes do IM que produziram mais de um (1) artigo, totalizando 34 professores analisados. Assim, chegou-se ao seguinte grafo: 
Grafo 1 - Principais colaborações entre autores.

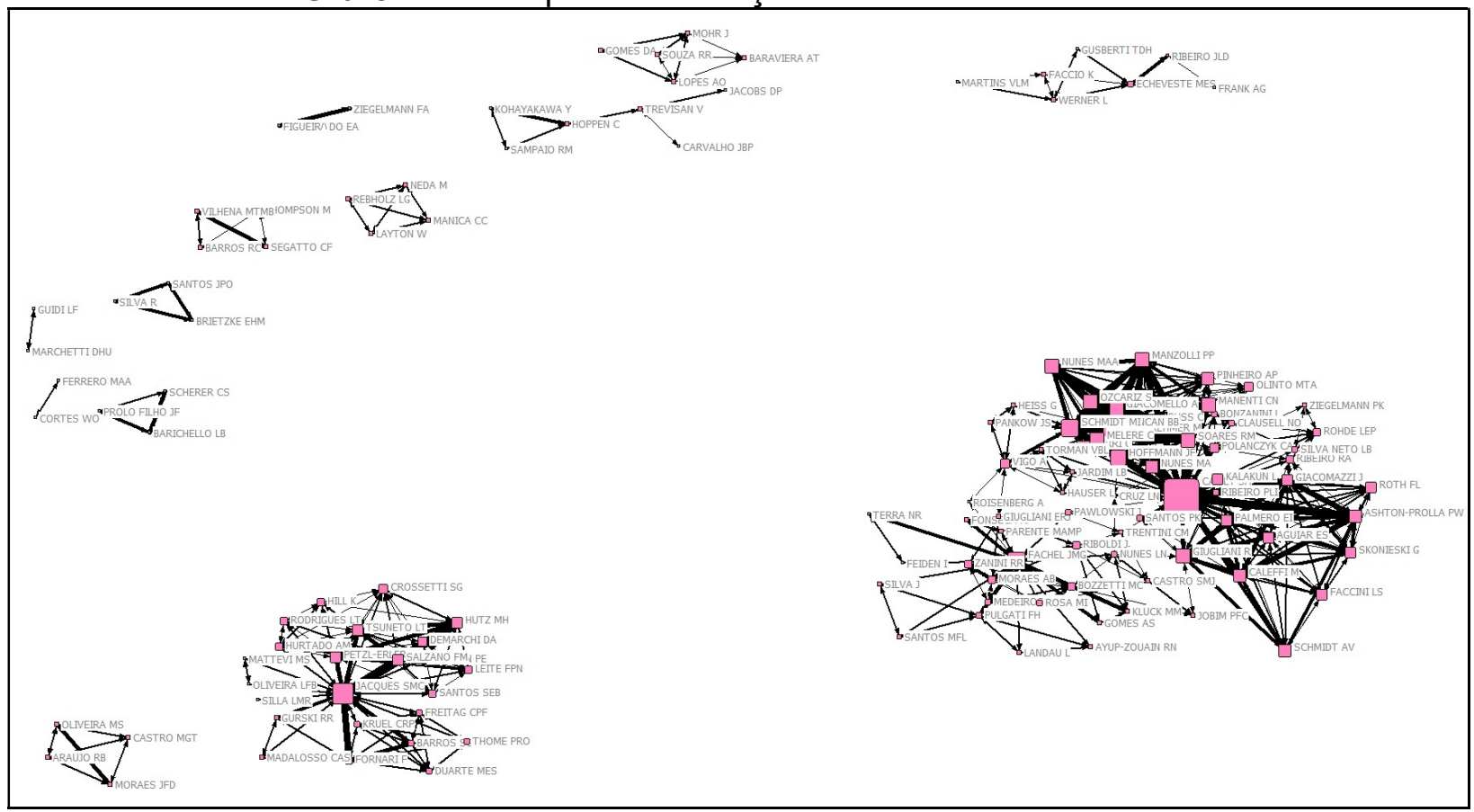

Fonte: Resultado da pesquisa.

A análise do grafo apresentado permite perceber a existência de 13 redes ou subgrupos, com 135 nós e 928 laços. Merece atenção o fato desses 13 subgrupos não estarem ligados por componentes externos. Além disso, também ganha destaque que 6 subgrupos são formados por docentes (BARICHELLO LB, BRIETZKE EHM, GUIDI LF, MANICA CC, MORAES JFD e ZIEGELMANN FA) os quais não colaboraram com seus colegas de instituição, formando redes isoladas de colaboração. A fim de entender o porquê da inexistência da coautoria intra ou mesmo interdepartamental desses docentes, investigou-se o Diretório de Grupo de Pesquisa, na Plataforma Lattes, para descobrir as áreas de interesse desses docentes.

MORAES JFD e ZIEGELMANN FA participam de grupos de pesquisa vinculados a outras instituições em que não fazem parte outros docentes do IM; MORAES JFD participa dos grupos Estatística Aplicada, IEPEC (Investigação sobre o Ensino de Probabilidade, Estatística e Combinatória), Grupo de Estudos e Pesquisa em Violência e Grupo de Avaliação e Atendimento em Psicoterapia Cognitiva e Comportamental, todos vinculados à PUCRS (Pontifícia Universidade Católica do Rio Grande do Sul), universidade em que é professor também; ZIEGELMANN FA participa do grupo Cópulas em Economia 
e Finanças, vinculado à Universidade Católica de Brasília (UCB), provavelmente porque um dos integrantes do grupo foi seu orientando no doutorado e hoje é docente da referida universidade. BARICHELLO LB faz parte do grupo Transporte de Partículas e Transferência Radiativa, o qual participa também mais um docente do IM, contudo, o referido docente esteve 7,5 anos na direção do Instituto, o que, provavelmente, o afastou da pesquisa. BRIETZKE EHM, que participa dos grupos Probabilidade e Análise Matemática (com 5 integrantes do IM) e Equações Diferenciais Parciais Elípticas (com 4 integrantes do IM) e GUIDI LF e MANICA CC, que participam do mesmo grupo - Algoritmos e Métodos Analíticos e Numéricos em Matemática Aplicada - com mais 5 pesquisadores do IM, não possuem em seus artigos coautoria intra ou interdepartamental. Cunha (2012) coloca que, apesar de, por natureza, a pesquisa em matemática possuir um caráter mais individual, há muita troca de informações entre os pesquisadores, contudo, não há considerável coautoria.

A partir do grafo anterior, com 13 redes, far-se-á a seguir a análise das quatro maiores e principais redes, por serem as mais colaborativas. A primeira rede a ser analisada é apresentada abaixo, com 69 atores e 640 laços. Nela está representada a EGONET (rede individual) de CAMEY SA (na cor roxa e em posição central nessa rede de coautoria). Pode-se considerar essa EGONET como a mais influente, já que apresenta o maior nó e em posição mais central (núcleo), indicando que CAMEY SA possui maior poder entre todos os nós existentes no grafo. CAMEY SA tem 42 laços, sugerindo que possui característica altamente colaborativa, em virtude do seu número expressivo de contatos com outros pesquisadores, inclusive do mesmo departamento em que trabalha (CASTRO SMJ, FACHEL JMG, NUNES LN, PULGATI FH, RIBOLDI J, TORMAN VBL, VIGO A e ZIEGELMANN PK). Geralmente os pesquisadores mais colaborativos são mais produtivos, o que vem ao encontro dos resultados encontrados aqui, em que CAMEY SA é a docente com a maior produção de artigos entre os anos de 2007 e 2011. Conforme Maltrás Barba (2003, p.242): 
[...] o aumento da colaboração [...] afeta de maneira direta o processo de geração de conhecimentos científicos, tanto em nível dos pesquisadores individuais como em nível das instituições e os recursos materiais colocados em jogo; e gera redes com efeitos coesivos entre os elementos que constituem a comunidade científica.

Grafo 2 - EGONET de CAMEY SA.

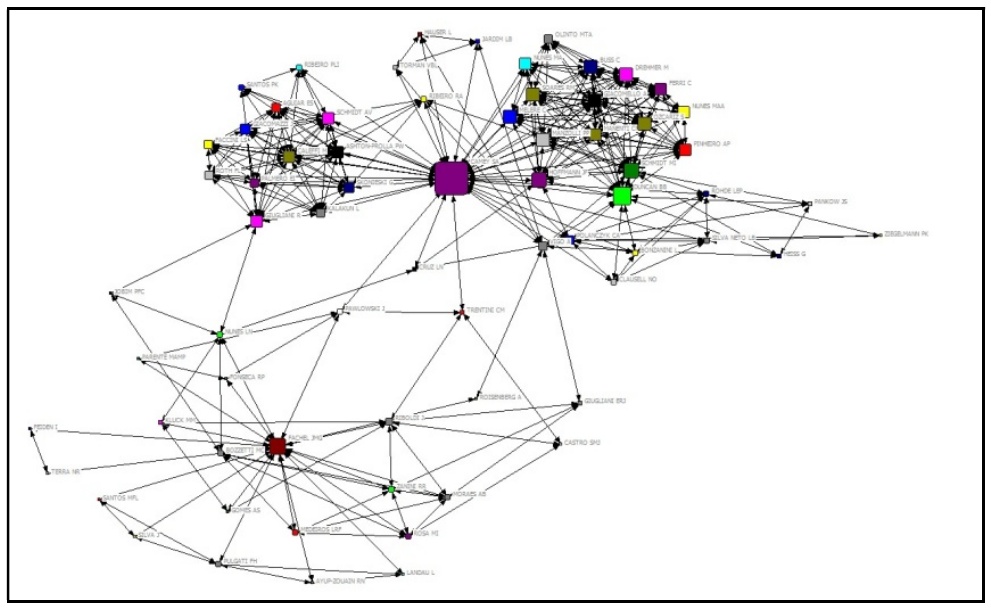

Fonte: Resultado da pesquisa

CAMEY SA tem o maior grau de intermediação (betweenness centrality) da rede: 1.265.940. Tal valor indica que a pesquisadora atua fortemente como ponte de conexão entre outros atores da rede. A pesquisadora apresentou grau de proximidade (closeness centrality) 9.018, o menor desse subgrupo, revelando a proximidade desse ator dos demais atores dessa rede.

A partir de uma análise mais profunda da rede em questão, nota-se que CAMEY SA possui laços mais fortes com ASHTON-PROLLA PW, CALEFFI M, MANZOLLI PP e MELERE C. Tais ligações mais fortes são decorrentes do grau de colaboração entre CAMEY SA e esses outros atores, indicando um envolvimento maior entre eles (relacionamento mais próximo), principalmente quando comparado com aqueles atores com ligações mais fracas; "a força com que se estabelecem determinadas relações, linhas mais largas significam maior colaboração" (COSTA, 2009, p.75). Assim, verificou-se que o relacionamento forte com ASHTON-PROLLA PW dá-se por meio de dois projetos de pesquisa em que trabalham juntas: "Consolidação de um Projeto Piloto de Identificação de Fatores de Risco para Câncer de Mama Hereditário de Base Populacional Vinculado ao Programa Saúde da Família" e "Estimativas de Risco de Câncer de Mama em uma População Submetida a Rastreamento Mamográfico em 
Porto Alegre" (neste, CALEFFI M também participa). CAMEY SA, da mesma forma, também compartilha do projeto de pesquisa "Medida do Padrão de Consumo Alimentar, Prevalência de Transtornos Mentais e Violência em uma Amostra de Gestantes" com MANZOLLI PP. E a relação dessa docente com MELERE C faz-se porque foi orientadora dessa pesquisadora em seu Mestrado em Epidemiologia/UFRGS, Programa de Pós-Graduação na qual CAMEY SA é docente.

No grafo também se pode perceber outros nós importantes, tais como DUNCAN BB, SCHMIDT MI, HOFFMANN JF e FACHEL JMG. DUNCAN BB faz parte do mesmo Grupo de Pesquisa em Análises Econômicas em Saúde que CAMEY SA, através do HCPA (Hospital de Clínicas de Porto Alegre); porque DUNCAN BB fez seu mestrado e seu pós-doutorado nos Estados Unidos, o que talvez explique o número de publicações de CAMEY SA nos Estados Unidos. Já a sua relação com SCHMIDT MI e com HOFFMANN JF dáse através dos projetos de pesquisa em que trabalharam juntas: "Projeto Colaborativo Interinstitucional de Estudos de Custo-Efetividade em Saúde" e "Medida do Padrão de Consumo Alimentar, Prevalência de Transtornos Mentais e Violência em uma Amostra de Gestantes", respectivamente.

FACHEL JMG (na cor vermelha e localizada à esquerda, embaixo) é um importante nó, não somente por participar do mesmo Grupo de Pesquisa Estatística Aplicada - que CAMEY SA, mas principalmente porque é a terceira docente do IM em importância dentro dessa rede analisada. Ela possui 20 laços, o que traduz característica colaborativa, até mesmo com seus colegas de departamento (CASTRO SMJ, NUNES LN, PULGATI FH, RIBOLDI J, TORMAN VBL, VIGO A e ZIEGELMANN PK). A docente possui alta produtividade, sendo a segunda professora que mais publicou artigos de periódicos no período analisado.

Em relação ao grau de intermediação, FACHEL JMG apresentou um grau de 614,24 , traduzindo a sua importância como ponte de conexão entre outros atores da rede. A docente apresentou o décimo menor grau de proximidade (9067), não se mostrando tão próxima assim do núcleo da rede, como até visualmente pode-se perceber ao analisar-se o grafo. 
Um exame minucioso permite verificar que FACHEL JMG tem laços mais fortes com KLUCK MM, em que foi orientadora de doutorado, e com PULGATI FH, seu colega de departamento, de Grupo de Pesquisa (Estatística Aplicada), projeto de pesquisa (Monitoramento Ambiental em Atividades de Perfuração Exploratória Marítima - Projeto MAPEM), coorientadora no doutorado e orientadora na graduação. Tais laços mais fortes são explicados pela quantidade de colaborações que resultaram em coautoria entre FACHEL JMG e esses outros dois pesquisadores.

Nessa rede chamam a atenção o grau de intermediação de outros nós. Assim, destaca-se PAWLOWSKI J, com grau de intermediação 401,62 (terceiro maior grau, menor apenas que o de CAMEY SA e FACHEL JMG); VIGO A, com 215,5; GIUGLIANI R, com 187,86 e NUNES LN, com 182,37. Interessante observar que esses quatro nós servem como ponte entre a sub-rede que se formou em torno de CAMEY SA (principal nó) e a sub-rede que se formou em torno de FACHEL JMG.

A segunda rede a ser analisada é apresentada abaixo, com 24 nós e 584 laços. Nela está representada a EGONET de JACQUES SMC (na cor vermelha e em posição central). Esta EGONET é a segunda mais importante, já que possui o segundo maior nó e em posição central. JACQUES SMC tem 23 laços, aventando que possui característica colaborativa, em decorrência do número de contatos que possui com outros pesquisadores. As colaborações de JACQUES SMC são todas inter e extradepartamental, ou seja, ela não tem colaborações com seus colegas do DEST. Provavelmente essa ausência de colaboração com seus colegas de departamento seja em virtude da pesquisadora possuir mestrado e doutorado na área de Genética e Biologia Molecular e, em consequência, seguir linhas de pesquisa muito diferentes da de seus colegas (Estrutura populacional e polimorfismos genéticos em índios sul-americanos: análise estatística). Em virtude dessa sua considerável colaboração, ela é a terceira docente mais produtiva. 
Grafo 3 - EGONET de JACQUES SMC

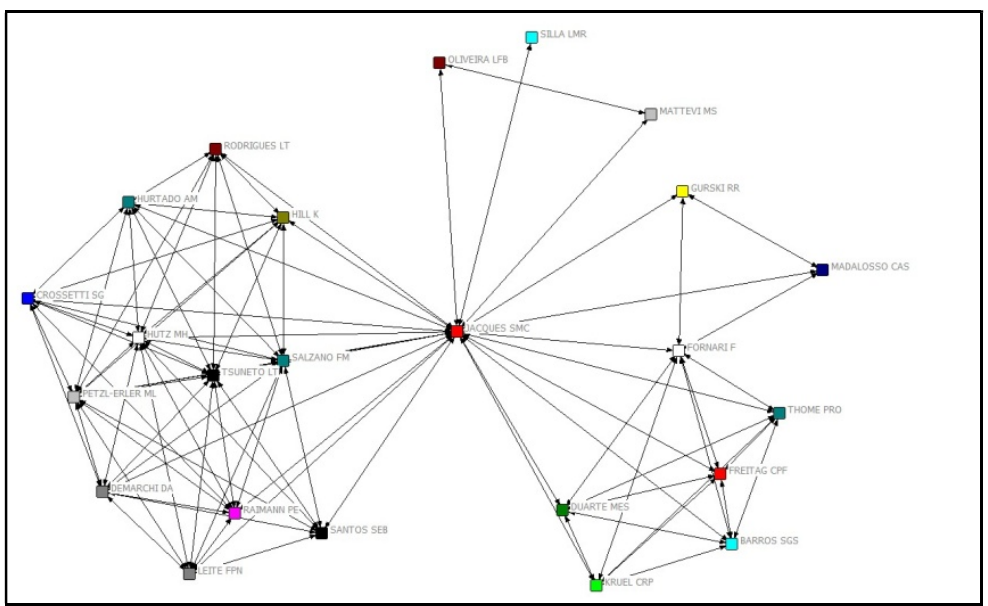

Fonte: Resultado da pesquisa.

JACQUES SMC tem o maior grau de intermediação da sua EGONET, com valor de 165,86. O segundo maior grau de intermediação nessa EGONET é de 2,8, mostrando que a pesquisadora é uma ponte de conexão entre atores da rede muito forte (como pode ser visualizado no grafo). O grau de proximidade de JACQUES SMC é de 15.008, sendo o menor da EGONET, ou seja, ela é a pesquisadora que possui maior proximidade com os demais atores da rede.

Analisando-se mais detalhadamente essa EGONET, nota-se que JACQUES SMC possui laços mais fortes com HUTZ MH e SALZANO FM. Tais ligações mais fortes revelam um grau de colaboração maior entre esses pesquisadores, os quais participam ou participaram dos mesmos projetos de pesquisa: "Análise da Variabilidade Genética em Humanos e em Outros Mamíferos Sul-Americanos" e "Estrutura Populacional em Populações Humanas da América do Sul: aplicações em genética histórica e farmacogenômica". Além disso, SALZANO FM foi seu orientador no mestrado e doutorado e, atualmente, é o líder do Grupo de Pesquisa - Genética de Populações Humanas e de Outros Organismos, da qual participa.

$\mathrm{Na}$ EGONET também se pode perceber outros nós importantes, tais como HUTZ MH, PETZL-ERLER ML, SALZANO FM e TSUNETO LT. No parágrafo anterior foram vistos os prováveis motivos de colaboração de JACQUES SMC e HUTZ MH e SALZANO FM. PETZL-ERLER ML e TSUNETO 
LT seguem a mesma linha de pesquisa, genética de populações, que JACQUES SMC, talvez estando aí o motivo de tantas colaborações.

Pelas análises feitas e baseando-se na teoria de Maltrás Barba (2003), infere-se que o critério de agregação de alguns pesquisadores seja o temático. O referido autor coloca que "os cientistas, unidos em um princípio de compartilhar objetivos e problemas de pesquisa, se relacionam para trocar informações e experiências sobre temas específicos" (MALTRÁS BARBA, 2003, p.78). Assim, formam-se as comunidades científicas.

\subsection{Colaboração Interinstitucional}

Nos 177 artigos de periódicos analisados, havia 530 autores, distribuídos em 110 instituições $^{6}$ (perfazendo uma média de 1,6 instituições por artigo); contudo 23 desses autores não estavam vinculados a instituição alguma (eram profissionais liberais). As seis instituições com mais autores são nacionais (UFRGS, DMPA, HCPA, DEST, HMV - Hospital Moinhos de Vento - e PUCRS) e nelas estão $55,66 \%$ dos autores (aqui se considerando o DEST e o DMPA como uma instituição cada um deles).

$\mathrm{Na}$ totalidade, entre os 507 autores com vínculo, 384 (75,73\%) são vinculados a instituições nacionais, enquanto que o restante, $123(24,26 \%)$, a instituições estrangeiras. Contudo, entre as 110 instituições, 64 (58,18\%) são estrangeiras e $46(41,81 \%)$, nacionais. Isso significa que se tem uma média de autores por instituição em nível nacional (8,34 autores) muito maior do que a média de autores por instituição em nível internacional (1,92 autores), significando que houve uma maior diversidade de instituições estrangeiras colaborando com os departamentos do IM.

6 Neste caso, foram desconsiderados os autores sem vínculo; para os autores que tinham mais de um vínculo no mesmo artigo, considerou-se somente o primeiro vínculo por ordem de aparição, com exceção daqueles autores que possuíam vínculo UFRGS e HCPA, em que considerou-se como sendo UFRGS. 
A partir dos vínculos dessas 110 instituições, gerou-se o grafo apresentado a seguir (contudo o grafo traz um nó SV, indicando aqueles autores "sem vínculo", totalizando em 111 instituições nesta situação).

\section{Grafo 6 - Colaboração entre instituições}

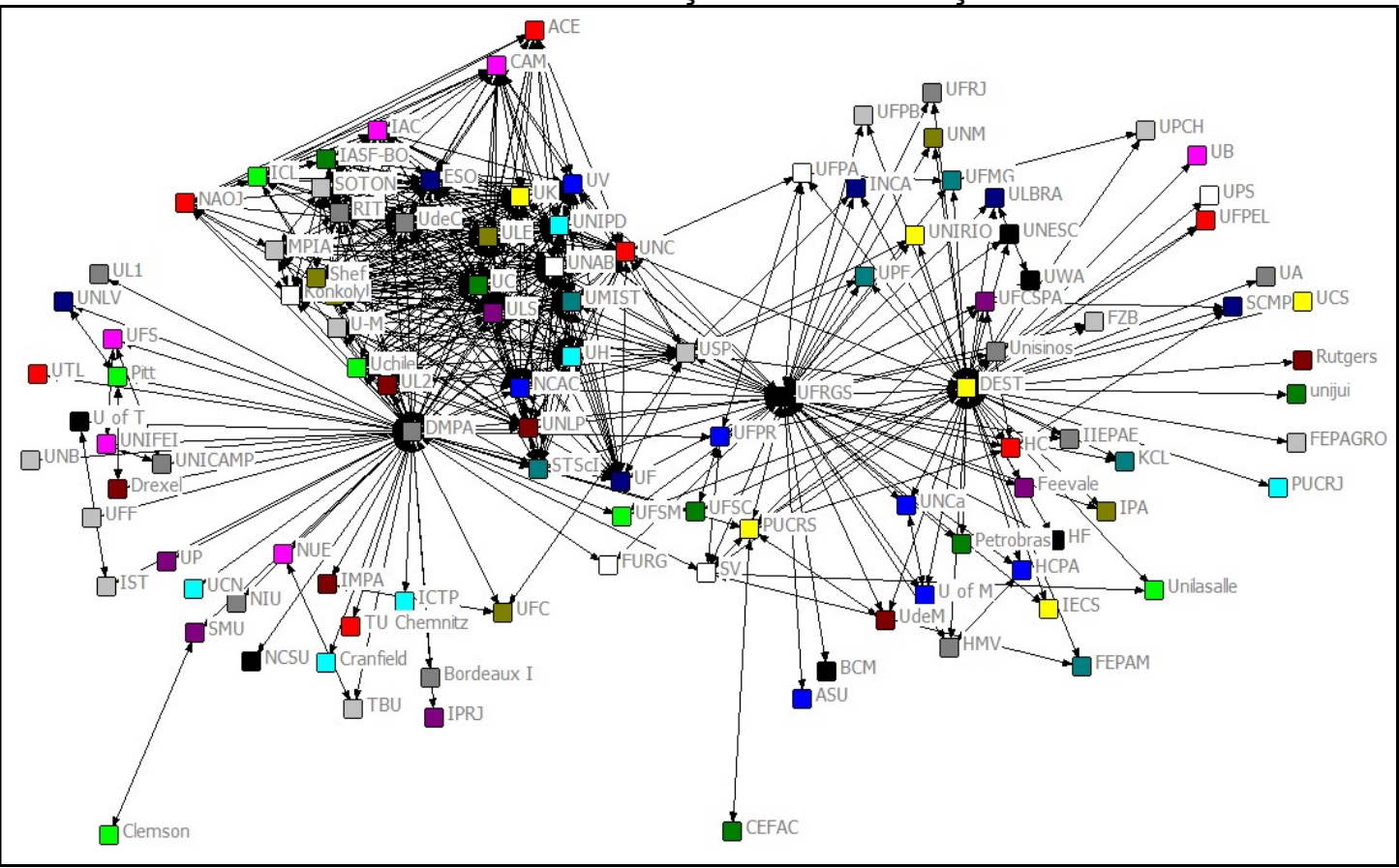

Fonte: Resultado da pesquisa

O grafo apresentado possui 111 nós e 980 laços, indicando uma forte colaboração interinstitucional. No entanto, vale destacar que inexistem linhas relacionais entre os departamentos DEST e DMPA mostrando que não há colaboração interdepartamental. Nesse grafo também se percebe a existência de três grandes redes (aglutinadas no meio do grafo, em virtude do seu grau de centralidade) - DMPA, DEST e UFRGS.

Em relação à UFRGS, muito importante destacar que este grafo foi gerado a partir das relações do DEST e do DMPA; logo, as relações que aparecem em torno da UFRGS são apenas parciais. Certamente a UFRGS possui relações de colaboração muito maiores do que as apresentadas aqui. Neste caso específico, a UFRGS apresenta 62 laços, sendo os mais densos com DEST, DMPA, HCPA, HMV, KCL (King's College London), UFPR (Universidade Federal do Paraná) e UNCa (University of North Carolina). Os laços existentes entre UFRGS e KCL, provavelmente, estejam explicados na 
relação de KCL com DEST, já que uma pesquisadora (FERRI C), coautora de artigos junto a CAMEY SA (DEST), fez seu pós-doutorado nessa instituição; ambas pesquisadoras fazem parte do Projeto de Pesquisa - Nutrição, Saúde Mental e Violência: da gravidez ao pós parto: coorte de mulheres atendendo pré-natal na rede primária em Porto Alegre - estudo ECCAGE. A ligação da UFRGS com a UNCa, muito provável que seja em função de que DUNCAN BB, docente da UFRGS, fez ali o seu pós-doutorado (esse professor colabora bastante com DEST). Pesquisadores da UFRGS e UFPR participam do mesmo Projeto de Pesquisa - Instituto do Milênio: ancestralidade genômica e identidade nacional implicações biomédicas e forenses.

A primeira rede a ser analisada é apresentada no Grafo 7, com 64 nós e 724 laços. Nela está representada a EGONET do DMPA (na cor cinza e em posição central dentro da rede). Pode-se considerar essa EGONET como a mais influente, já que apresenta o maior nó e está em posição mais central, indicando que o DMPA possui maior poder entre todos os nós existentes na rede. O DMPA tem 63 laços, sendo a instituição mais colaborativa de toda a rede. Contudo o DMPA não foi a instituição com maior produtividade.

\section{Grafo 7 - EGONET do DMPA.}

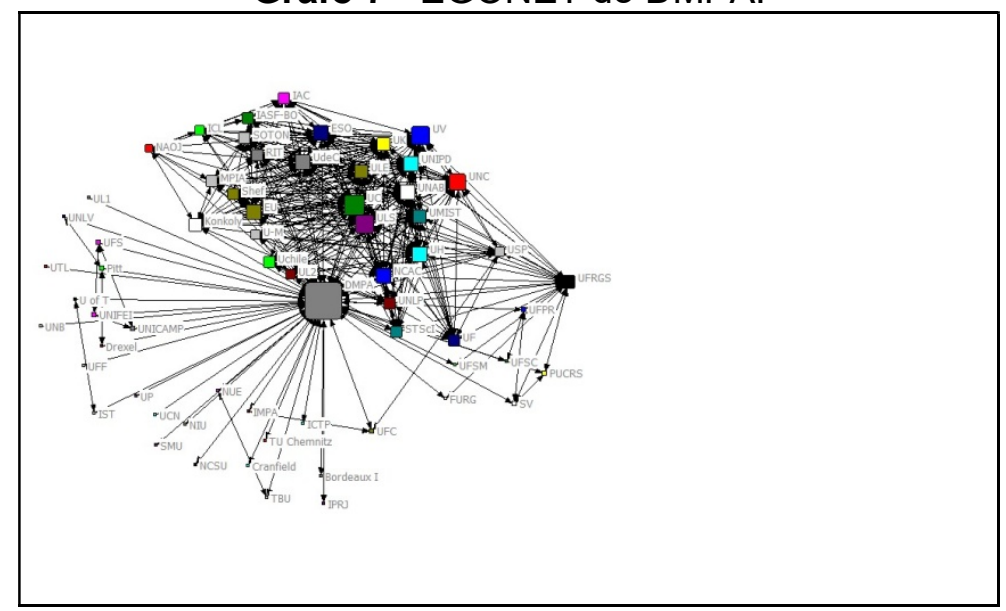

Fonte: Resultado da pesquisa

O DMPA tem o maior grau de intermediação do grafo: 2.828.703. Tal valor indica que a instituição é uma forte ponte de conexão entre as outras instituições da rede. $\mathrm{O}$ referido departamento apresentou grau de proximidade 
168.000, o menor do grafo, revelando a proximidade do DMPA com os demais nós da rede.

A partir de uma análise mais profunda dessa rede, nota-se que o DMPA possui laços mais fortes com UFRGS e UC (Pontificia Universidad Católica de Chile). Tais ligações mais fortes são decorrentes de um maior grau de colaboração entre DMPA e essas outras instituições, indicando um relacionamento mais próximo.

DMPA e UFRGS possuem laços mais fortes em virtude de seus pesquisadores, por exemplo, participarem dos mesmos Grupos de Pesquisa (Grupo em Teoria de Transporte de Partículas e Transformadas Integrais; Transporte de Partículas e Transferência Radiativa), junto a outras unidades da UFRGS, tais como a Escola de Engenharia. Também por possuírem em comum Projetos de Pesquisa ( $\mathrm{O}$ Controle da Deriva Litorânea no Desenvolvimento do Campo de Dunas e da Antepraia no Litoral do RS; Monitoramento do Campo Ondulatório em Tramandaí - RS; Desenvolvimento de Soluções de Caráter Analítico e Estudos de Aspectos Computacionais para Modelos de Transporte de Partículas em Geometrias Multidimensionais; Desenvolvimento de Soluções de Caráter Analítico e Estudo de Aspectos Computacionais para Modelos da Dinâmica de Gases Rarefeitos e Transferência Radiativa; Avaliação de Tecnologias Educacionais), em conjunto também com outras unidades da UFRGS, como o Instituto de Geociências e o Instituto de Informática. E, da mesma forma, pelas orientações de teses, dissertações e trabalhos de conclusão de curso.

Já DMPA e UC possuem vínculos mais fortes em virtude de um único artigo de periódico publicado em comum, na área da física, subárea da astronomia. O referido artigo possui, entre seus autores, dez coautores vinculados a essa universidade do Chile.

$\mathrm{Na}$ rede também se pode perceber outros nós importantes, tais como UC, ULS (Universidad de La Serena), UV (Universidad de Valparaíso), UNC (Universidad Nacional de Córdoba), UdeC (Universidad de Concepción) e UNIPD (Universitá di Padova). Os motivos pelos quais essas instituições são nós importantes dentro da rede, da mesma forma como colocado em parágrafo 
anterior, giram em torno do artigo publicado a respeito do tema astronomia. Artigo esse que conta com 66 autores, estando aí o motivo da diversidade de instituições vinculadas.

Nesta rede é importante destacar o número de instituições estrangeiras e nacionais. DMPA relaciona-se com 45 (72,58\%) instituições estrangeiras e 17 $(27,41 \%)$ instituições nacionais (desconsiderando-se os "sem vínculo"). Diante desses dados, percebe-se que há um número muito maior de instituições estrangeiras vinculadas ao DMPA. O motivo desse cenário estaria no fato de que os docentes do DMPA saem muito para o exterior para dar continuidade aos estudos de pós-graduação, dessa forma, incrementando as parcerias com instituições estrangeiras. Vanz (2009) também percebe esse aumento na parceria estrangeira, colocando que programas tais como bolsa-sanduíche, ofertada pela CAPES e CNPq (em que o estudante faz uma parte de seu pósgraduação no exterior), e estágio pós-doutoral, oferecida pela CAPES, são formas de se criar vínculos com instituições estrangeiras.

A segunda rede a ser analisada é apresentada no Grafo 8, com 50 nós e 214 laços. Nela está representada a EGONET do DEST (na cor amarela e em posição mais central dentro da rede). Pode-se considerar essa EGONET como a terceira mais influente, já que apresenta o terceiro maior nó e está localizada em posição mais central, indicando que também possui poder dentro da rede. A rede do DEST apresenta 48 laços, sendo a instituição com maior produtividade nesta pesquisa.

Grafo 8 - EGONET do DEST.

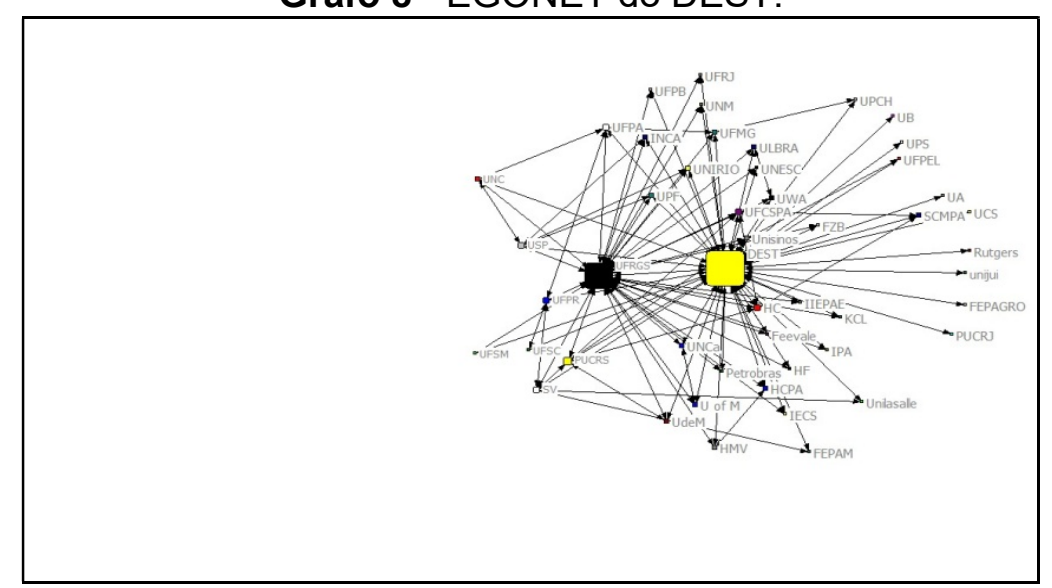

Fonte: Resultado da pesquisa 
O DEST tem o terceiro maior grau de intermediação do grafo: 1.533.842. Esse valor designa que tal instituição também é uma ponte de conexão forte entre os demais nós da rede. O seu grau de proximidade é de 201.000 , sendo o quarto menor do grafo, revelando a sua proximidade com as demais instituições, mas ficando atrás do DMPA, UFRGS e UNC.

Uma análise mais minuciosa na rede permite que se encontrem laços mais fortes entre o DEST e FEPAM (Fundação Estadual de Proteção Ambiental Henrique Luis Roessler-Rio Grande do Sul), HCPA, HMV, PUCRS, UFRGS e UFSM (Universidade Federal de Santa Maria). Esses laços decorrem da maior colaboração entre essas instituições, revelando um relacionamento mais direto.

DEST e FEPAM têm laços mais fortes porque FACHEL JMG participa de dois Grupos de Pesquisa - Geoquímica ambiental e Ecotoxicologia: Genética Toxicológica, cuja coordenação é da FEPAM. DEST e HCPA possuem laços fortes em decorrência de alguns Grupos de Pesquisa, aos quais determinados docentes do DEST fazem parte: Análises Econômicas em Saúde (vinculado ao próprio HCPA); Epidemiologia do Diabetes e das Doenças Cardiovasculares (ligado ao Departamento de Medicina Social, da Faculdade de Medicina/UFRGS) e Genética de Populações Humanas e de Outros Organismos (vinculado ao Departamento de Genética, do Instituto de Biociências/UFRGS). Além disso, existem Projetos de Pesquisa em comum entre as duas instituições: Um Estudo Prospectivo da Doença de MachadoJoseph e Estudo da Dinâmica de Transmissão de Infecção em um Surto de Acinetobacter Baumanii no Hospital de Clínicas de Porto Alegre.

Os laços entre DEST e HMV decorrem de Projetos de Pesquisa (“Consolidação de um Projeto Piloto de Identificação de Fatores de Risco para Câncer de Mama Hereditário de Base Populacional Vinculado ao Programa Saúde da Família", "Estimativas de Risco de Câncer de Mama em uma População Submetida a Rastreamento Mamográfico em Porto Alegre" e “Identificação e Caracterização de Pacientes em Risco para Câncer de Mama Hereditário Provenientes de uma Amostra Populacional do Sul do Brasil”), todos ligados ao estudo do câncer, foco de diversos pesquisadores ligados ao HMV. A relação forte entre DEST e PUCRS dá-se em função, provavelmente, 
porque dois docentes do DEST realizaram especialização na PUCRS, criando laço de colaboração; além disso, dois professores do DEST também são docentes no Departamento de Estatística da PUCRS.

Os laços entre DEST e UFRGS ocorrem principalmente com a Faculdade de Medicina, Escola de Engenharia, Instituto de Química, Instituto de Geociências, Instituto de Biociências, Instituto de Psicologia e Faculdade de Veterinária. A ligação do DEST com UFSM está baseada, provavelmente, no fato de um de seus professores ter trabalhado lá e um outro docente ter realizado a sua graduação lá também; além disso, na UFSM há um docente que já trabalhou no DEST.

Da mesma forma, na rede também se pode notar outros importantes nós, tais como UFRGS, PUCRS, SV e USP (Universidade de São Paulo). UFRGS e PUCRS já foram analisadas aqui. SV diz respeito àqueles pesquisadores sem vínculos com instituições de ensino e pesquisa, ou seja, não se podem fazer maiores inferências a respeito. Tudo indica que USP é um importante nó em virtude de diversos de seus professores (9 no total) terem sido vinculados aos programas de pós-graduação da universidade em questão, promovendo laços substanciais de colaboração.

Nesta rede é importante destacar que o número de instituições nacionais é maior do que o de instituições estrangeiras (ao contrário do DMPA). DEST está relacionado com 14 (29,16\%) instituições estrangeiras e 34 (70,83\%) instituições nacionais, não se considerando os "sem vínculo". A explicação para tal fato pode estar atrelada ao fato de que os docentes do DEST maciçamente fizeram suas pós-graduações no Brasil, dessa forma, estreitando relações com as instituições nacionais.

\section{CONSIDERAÇÕES FINAIS}

Esse estudo teve por objetivo avaliar a produção docente dos Departamentos de Estatística e de Matemática Pura e Aplicada, do Instituto de Matemática/UFRGS, em relação aos artigos de periódicos publicados eletronicamente, entre os anos de 2007 e 2011. Além disso, foram objetivos 
também a análise da produtividade de artigos publicados no período por docente, a verificação do Qualis e da nacionalidade dos periódicos e a avaliação do nível de colaboração entre os autores e entre as instituições.

A produtividade de artigos publicados no período por docente trouxe como resultado 177 artigos na totalidade, sendo que em 32 deles mais de um docente do IM participou. Dos 89 docentes da Unidade, 53 publicaram artigos no período analisado, contra 36 que nada produziram. Nesse sentido cabe aqui um novo estudo, a fim de se entender as causas pelas quais $40,5 \%$ dos docentes nada produziram nesses cinco anos, sendo que a produção intelectual docente traz visibilidade, promoções na carreira e financiamentos para pesquisas. Talvez isto possa ser explicado pelo fato destes pesquisadores terem publicado em periódicos e esta produção não foi informada à Biblioteca do IM, para que a produção seja inserida no SABi.

Nos resultados encontrados chama a atenção que apenas sete docentes sejam responsáveis por $48,59 \%$ da produção de artigos do IM. Para tal fato, encontrou-se embasamento teórico, na Lei do Elitismo de Price e na Lei de Produtividade de Lotka. Também merece destaque a superioridade da produção do DEST em relação ao DMPA, apesar do número menor de docentes no departamento, cujas causas devem ser avaliadas em estudos qualitativos, assim como sugere-se um estudo para entendimento do aumento substancial na produção como um todo a partir do ano de 2009.

A verificação do Qualis foi feita para 125 periódicos, muitos dos quais receberam mais de uma classificação, pois abrangiam mais de uma área do conhecimento. A grande maioria dos periódicos recebeu classificação B1, seguida da B2 e A2. Interessante observar que o resultado encontrado aqui para produtividade versus qualidade, confirmou a teoria de Meadows (1999), a respeito dos quatro tipos de pesquisadores, já que entre 11 docentes que mais publicam periódicos com alto Qualis, 7 estão no rol daqueles que mais produzem artigos; 4 docentes que publicam nos periódicos de melhor Qualis não estão entre aqueles com maior número de produção de artigos; 5 docentes com maior produção de artigos não se encontram entre aqueles que publicam em periódicos com maior Qualis. 
A verificação da nacionalidade dos periódicos apontou para um grande número de periódicos estrangeiros (62\%) em contrapartida aos nacionais (38\%). E um exame mais minucioso a respeito das instituições aos quais os coautores dos artigos estão vinculados (73,9\% dos autores estão ligados a instituições de ensino e pesquisa nacionais) levou-se a pensar que, felizmente, a pesquisa realizada pelos docentes do IM está atingindo o âmbito internacional.

A avaliação do nível de colaboração entre os autores permitiu que se concluísse que tal nível é bastante elevado em função do número de coautorias que se encontrou (somente 4,5\% dos artigos eram de autoria individual), indo ao encontro da tese de Vanz (2009). Chama a atenção as 13 redes formadas no Grafo 2, pois elas não se comunicam, mostrando que no IM há diversas linhas de pesquisa heterogêneas, comprovando que a estatística e a matemática servem também como base para o estudo em outras ciências. Vale destacar que foi fundamental a investigação nos Grupos de Pesquisa e nos Projetos de Pesquisa publicados no Lattes para se entender como se deram algumas relações de colaboração. Cabe aqui também um estudo mais aprofundado com o objetivo de entender melhor a alta produtividade e a alta colaboração das três docentes do DEST: CAMEY SA, JACQUES SMC e FACHEL JMG, as quais apresentam números superiores aos dos seus colegas de unidade.

A avaliação do nível de colaboração entre as instituições permitiu que se verificasse que há um bom nível de relações entre DEST e outras instituições e entre DMPA e outras instituições de pesquisa e ensino (108 no total); mas que não há colaboração entre DEST e DMPA, apesar de serem departamentos pertencentes ao mesmo Instituto. Esses laços mais fortes de colaboração se dão, principalmente, entre instituições brasileiras; contudo, há uma maior variedade de instituições estrangeiras vinculadas $(58,18 \%)$. Esse expressivo número de intercâmbio de conhecimento com instituições estrangeiras faz pensar que o Brasil está reconhecidamente fazendo pesquisa em excelência. Da mesma forma em relação aos autores dos artigos, a pesquisa nos Grupos de Pesquisa do Lattes fez entender o relacionamento entre diversas 
instituições, dando destaque para a grande afinidade que o DEST tem com instituições da área da saúde.

As áreas da Estatística e da Matemática não são comparáveis, pois são áreas distintas em se tratando de práticas comunicacionais. Com esse estudo, então, pretendeu-se mostrar o cenário de produção do $\mathrm{IM}$ e, em nenhum momento, intencionou-se que esse estudo trouxesse respostas conclusivas.

\section{REFERÊNCIAS}

ARAÚJO, Carlos Alberto. Bibliometria: evolução histórica e questões atuais. Em Questão, Porto Alegre, v. 12, n. 1, p. 11-32, jan./jun. 2006. Semestral. Disponível em: <http://revistas.univerciencia.org/index.php/revistaemquestao/article/view/3707/ 3495>. Acesso em: 13 out. 2011.

BRASIL. Decreto $n^{\circ}$ 6.096, de 24 de abril de 2007. Institui o Programa de Apoio a Planos de Reestruturação e Expansão das Universidades Federais - REUNI. Diário Oficial da União, Brasília, DF, 24 abr. 2007. Disponível em: $<$ https://www.planalto.gov.br/ccivil 03/ ato2007-

2010/2007/decreto/d6096.htm>. Acesso em: 2 jul. 2012.

COSTA, Josiane Gonçalves da. A Produção Intelectual Docente do

Departamento de Ciências da Informação da UFRGS: estudo bibliométrico. 2009. 115 f. Trabalho de Conclusão de Curso (Especialização) - Curso de Especialização em Gestão de Bibliotecas Universitárias, Faculdade de Biblioteconomia e Comunicação, UFRGS, Porto Alegre, 2009. Disponível em: <http://www.lume.ufrgs.br/bitstream/handle/10183/18491/000730121.pdf?seque nce=>. Acesso em: 11 out. 2011.

CUNHA, Rudnei Dias da. Rudnei Dias da Cunha: depoimento em 1 de jun. 2012. Entrevistadora: Daniela Gralha de Caneda Queiroz. Porto Alegre, 2012. Anotações pessoais.

CRUZ, Fernanda. Brasil está em $14^{\circ}$ lugar no ranking mundial de pesquisas científicas. Agência Brasil, Brasília, 17 set. 2013. Pesquisa e Opinião. Disponível em: <http://memoria.ebc.com.br/agenciabrasil/noticia/2013-0917/brasil-esta-em-14\%C2\%BA-lugar-no-ranking-mundial-de-pesquisascientificas>. Acesso em: 22 jan. 2014.

MALTRÁS BARBA, Bruno. Los Indicadores Bibliométricos: fundamentos y aplicación al análisis de la ciencia. Gijón: Trea, 2003. 288 p.

MEADOWS, Arthur Jack. A Comunicação Científica. Trad. Antônio Agenor Briquet de Lemos. Brasília, DF: Briquet de Lemos, 1999. 268 p. 
MENEGHINI, Rogerio. Inusitado aumento da produção científica. Folha de S. Paulo, São Paulo, 12 maio 2009. Opinião, p. A3. Disponível em: <http://acervo.folha.com.br/fsp/2009/05/12/2/5325903>. Acesso em: 24 nov. 2011.

THOMAZ, Petronio Generoso; ASSAD, Renato Samy; MOREIRA, Luiz Felipe $P$. Uso do fator de impacto e do índice $\mathrm{H}$ para avaliar pesquisadores e publicações. Arquivos Brasileiros de Cardiologia, São Paulo, v. 96, n.2, p. 90-93, fev. 2011. Disponível em: <http://dx.doi.org/10.1590/S0066782X2011000200001>. Acesso em: 3 jun. 2012.

UNIVERSIDADE FEDERAL DE SÃO PAULO. Fator de Impacto, 2012. São Paulo, 2012. Disponível em: <http://bc.bireme.br/f impacto.htm>. Acesso em 3 jun. 2012.

VANZ, Samile Andréa de Souza. As Redes de Colaboração Científica no Brasil: 2004-2006. 2009. 204 f. Tese (Doutorado) - Programa de PósGraduação em Comunicação e Informação, Faculdade de Biblioteconomia e Comunicação, UFRGS, Porto Alegre, 2009. Disponível em: $<$ http://www.lume.ufrgs.br/bitstream/handle/10183/17169/000711634.pdf?seque nce=1>. Acesso em: 11 out. 2011.

\title{
Title
}

Teaching intellectual production of the math institute/ufgrs

\begin{abstract}
:
Introduction: The present study is a bibliometric research whose purpose is the evaluation of teaching production of the Statistics Department (DEST) and the Pure and Applied Math Department (DMPA) of the Math Institute (IM) of the Federal University of Rio Grande do Sul (UFRGS), concerning of the articles published only in web journals between 2007 and 2011. Objective: The specific goals of this study are: an individual analysis of productivity of the articles by author, a verification of the Qualis and the origin of the journal and an evaluation of the level of collaboration among authors and institutions.

Methodology: It's a study of bibliometrics with a qualitative approach using a questionnaire, and the answers were analyzed in the form of graphics, tables and others. Results: The results show 177 articles, and DEST $(64,4 \%)$ has a bigger production than DMPA (35,6\%). Most of the Qualis of the articles are B1 $(27,11 \%)$, B2 $(16,38 \%)$ e $A 2(15,81 \%)$. Foreign journals (62\%) appears more than Brazilian (38\%). Conclusion: The study shows that IM/UFRGS teachers are part of an impressive academic network. There's a good level of collaboration, most of the part with national institutions. The results also suggests new studies to find unanswered questions.
\end{abstract}

Keywords: Bibliometrics. Production teacher. Journals. Co-authorship. Interinstitutional collaboration. 


\section{Titulo}

Producción intelectual de los profesores del instituto de matemática/ufrgs

\section{Resumen:}

Introducción: Este estudio es una investigación bibliométrica cuyo objetivo es evaluar la producción de los profesores del Departamento de Estadística (DEST) e del Departamento de Matemática Pura y Aplicada (DMA) del Instituto de Matemática de la Universidad Federal del Rio Grande do Sul (IM/UFRGS) acerca de los artículos publicados en medio electrónico entre los años de 2007 y 2011. Objetivo: Los objetivos específicos son analizar la productividad de los artículos pro profesor, verificar el Qualis y la nacionalidad de los periódicos y por fin evaluar el nivel de colaboración entre los articulistas y las instituciones. Metodología: Es un estudio descriptivo bibliométrico con un abordaje cuantitativo mediante cuestionarios con sus respuestas analizadas con utilización de gráficos, tablas y cuadros. Resultados: Presenta 177 artículos donde la producción de DEST $(64,4 \%)$ es mayor que de DMA $(35,6 \%)$. Los Qualis en los periódicos analizados son en su mayoría B1 (27,11\%), B2 $(16,38 \%)$ y A2 $(15,81 \%)$. También presenta que los periódicos son en su mayoría extranjeros (62\%), y la menor parte nacionales (38\%). Conclusión: Esclarece que los profesores de IM/UFRGS tienen alto nivel de colaboración y una densa rede de relacionamiento. Hay un buen nivel de colaboración con otras instituciones en su mayoría nacionales. Resta una sugestión de nuevos estudios en el futuro para esclarecer las cuestiones sin respuesta.

Palabras clave: Bibliometría. Producción de los profesores. Periódicos. Coautor. Colaboración interinstitucional.

Recebido em: 19.11.2014

Aceito em: 05.12.2017 